\title{
EDITORIALS
}

\section{Filling the Black Hole of Hospital Discharge (Editorial in Response to Article by Walz et al., J Gen Intern Med 2011)}

\author{
Robert M. Wachter, MD and Andrew D. Auerbach, MD, MPH \\ Department of Medicine, University of California, San Francisco, San Francisco, CA, USA.
}

$\mathrm{J}$ Gen Intern Med 26(4):354-5

DOI: $10.1007 / \mathrm{s} 11606-011-1643-7$

(c) Society of General Internal Medicine 2011

$\mathrm{E}$ vidence of poor transitions in American health care is so widespread that data illustrating additional gaps have nearly lost their capacity to shock. Just consider these figures: nearly 20 percent of patients experience adverse events within 3 weeks of discharge ${ }^{1}$; nearly one in three heart failure patients is readmitted within a month after hospital discharge $^{2}$; and three out of four post-discharge follow-up visits occur without the benefit of a discharge summary ${ }^{3}$.

In this issue of JGIM, Walz and colleagues ${ }^{4}$ add another scary data point to this collection of statistics. Among 564 patients discharged from a large academic medical center to a sub-acute care facility, 181 (32\%) had pending laboratory tests, usually results of urine and blood cultures. Of these, only 20 $(11 \%)$ of the discharge summaries provided documentation of these pending tests. While prior studies ${ }^{5,6}$ have reported relatively poor documentation of tests pending at discharge, this study is the first to examine a population of patients being discharged to another facility-a situation in which one might expect documentation to be stronger since there is a receiving institution. With the publication of this paper, the black hole of hospital discharge becomes a little inkier.

As Batalden once observed, every system is perfectly designed to achieve the results it gets, and the hospital discharge process is clearly one such non-system. Responsibility for educating the patient and organizing a coherent discharge plan is diffuse, and often nonexistent. Hospital information systems are separated from outpatient or subacute care facility systems. Inpatient and outpatient providers frequently don't speak to each other. The result: it is often left up to an anxious and frail patient, or his or her family, to navigate these choppy post-hospital waters.

This is a failure of systems, but it also a failure of payment policy and of accreditation and regulatory standards. The hospital that receives its full payment regardless of the quality of its discharge communication or its readmission rate will focus on managing the hospitalization well but hesitate to pour resources into improving its discharge process. The same can be said for the involved providers, both inpatient and outpatient. The lax standards of the Joint Commission regarding discharge summaries further aggravates the prob$1 \mathrm{em}^{7,8}$. The Joint Commission requires that a discharge

Published online February 15, 2011 summary be completed within 30 days of discharge, and there is no mandate to document pending tests, let alone ensure that the summary is received by the follow-up provider.

Moreover, makers of information technology systems have lacked an incentive to create systems that can "speak" to each other. This means that unless the hospital and nursing home happen to be part of the same organization and are using the same IT platform, there is a strong chance that computerized evidence of pending tests-whether in the discharge summary or not-will not be transmitted to the follow-up provider.

Luckily, this is all beginning to change. In 2009 Medicare began publicly reporting hospitals' 30-day readmission rates for common diseases. In the next few years, it will begin penalizing hospitals with excess numbers of readmissions. This pressure has helped catalyze a variety of innovative programs that have been demonstrated to be effective in preventing readmissions, such as the Care Transitions Initiative $^{9}$, Project Red ${ }^{10}$, and Project BOOST ${ }^{11}$. The federal government is using the carrot of its stimulus funds to promote meaningful use standards, which require increasing levels of interoperability across IT platforms ${ }^{12}$.

But more needs to be done. The Joint Commission and other accreditors should be more aggressive about improving the discharge process. As one example, an accreditation standard that required hospitals to ensure that complete discharge summaries are on the desks of follow-up providers within 48 $\mathrm{h}$ of discharge would rapidly improve the situation. In our wired world, this is easily achievable, given resources and will.

But the mere presence of a discharge summary isn't enough. The summary needs to contain the right content, including a clear listing of pending tests. And even that may not be enough, since Dalal et al. have shown that making available a computerized list of pending tests to the ambulatory provider is no guarantee that the tests will be followed up ${ }^{13}$. Instead, information technology systems should be organized such that pending tests trigger provider alerts and populate task lists both in the hospital and the next setting of care, whether it is a nursing home or an ambulatory practice.

But even if we could create systems that appropriately chronicled pending tests and moved this information to the right places at the right times, there is a larger question: whose responsibility is it to follow up on tests pending at the time of discharge? There are two ways to conceptualize this. In one, the inpatient provider completes his or her tasks at the time of discharge, and the responsibility falls to the next provider to address any pending test results. Under this model, the inpatient provider (these days, generally a hospitalist) and his/her system are accountable for ensuring a good handoff to the follow-up provider and system. In the alternative model, the inpatient provider retains responsibility for following up on any 
test he or she sent, until all tests have returned or the patient has made formal contact with the follow-up provider (i.e., through a clinic visit).

We generally favor the first model, since some tests take days, even weeks, to return, during which time many hospitalists will rotate "off service" or even go on vacation, thus mandating yet another inpatient handoff if the hospitalist system remains responsible for follow-up. Moreover, some test results will require outpatient action (e.g., a repeat culture, the initiation of antibiotics), action that the outpatient provider is better positioned to orchestrate. We particularly favor the first model when patients are discharged to sub-acute care institutions, since there is a physician in this setting (such as the skilled nursing facility's medical director) who can be tasked with ensuring follow-up.

While we prefer the first model overall, our belief is that either model can work in different settings. The key is that each practice establishes unambiguous standards that clearly articulate who is responsible for what, supported by appropriate policies and IT tools ${ }^{3}$. Whichever standard is chosen, it is the hospitalists' and the hospital's responsibility to ensure that they follow the protocol set out for them-either by following up on the test itself or by communicating the need to follow up to the next system/provider.

While studies like the one by Walz and colleagues are helping to fill in the gaps in our knowledge about how to manage tests pending at discharge, we have much more to learn. Are certain pending tests more or less dangerous for the patient? Perhaps certain tests should not be ordered in the hospital but deferred to the outpatient setting. Is there a role for short-term followup, such as through a discharge clinic $^{14}$ or telemedicine models ${ }^{15}$, to ensure timely follow-up of key issues and tests that emerged during the hospitalization? Knowing the answers to questions like these will help us design the optimal discharge system for all inpatients.

Walz and colleagues never tell us how many of the tests pending at discharge returned with abnormal results, or whether patients were harmed by the poor information transfer. Perhaps few were. But one is too many. How can it be that in 2011, in a health care system that consumes nearly $\$ 3$ trillion per year, we cannot reliably perform the simple act of communicating important, potentially life-saving information from a hospital to another caregiver and facility? Let's get this fixed.
Corresponding Author: Robert M. Wachter, MD; Department of Medicine, University of California, San Francisco, Room M-994, 505 Parnassus Avenue, San Francisco, CA 94143-0120, USA (e-mail: bobw@medicine.ucsf.edu).

\section{REFERENCES}

1. Forster AJ, Murff HJ, Peterson JF, Gandhi TK, Bates DW. The incidence and severity of adverse events affecting patients after discharge from the hospital. Ann Intern Med. 2003;138:161-7.

2. Jencks SF, Williams MV, Coleman EA. Rehospitalizations among patients in the Medicare fee-for-service program. N Engl J Med. 2009;360: 1418-28.

3. Kripalani S, LeFevre F, Phillips Co, et al. Deficits in communication and information transfer between hospital-based and primary care physicians: Implications for patient safety and continuity of care. JAMA. 2007;297:831-41.

4. Walz SE, Smith M, Cox E, Sattin J, Kind AJH. Pending laboratory tests and the hospital discharge summary in patients discharged to sub-acute care. J Gen Intern Med 2011; doi:10.1007/s11606-010-1583-7.

5. Roy CL, Poon EG, Karson AS, et al. Patient safety concerns arising from test results that return after hospital discharge. Ann Intern Med. 2005; 143:121-8.

6. Were MC, Li X, Kesterson J, Cadwallader J, Asirwa C, Khan B, Rosenman MB. Adequacy of hospital discharge summaries in documenting tests with pending results and outpatient follow-up providers. J Gen Intern Med. 2009;24:1002-6.

7. Standard IM.6.10. Hospital Accreditation Standards. Oakbrook Terrace, IL: Joint Commission Resources; 2006:338-340

8. Kind AJ, Smith MA. Documentation of mandated discharge summary components in transitions from acute to subacute care. In Henriksen K, Battles JB, Keyes MA, et al., editors. Advances in Patient Safety: New Directions and Alternative Approaches (Vol. 2: Culture and Redesign). Rockville, MD: Agency for Healthcare Research and Quality, 2008

9. Coleman EA, Parry C, Chalmers S, Min SJ. The care transitions intervention: results of a randomized controlled trial. Arch Intern Med. 2006; 166:1822-8.

10. Jack BW, Chetty VK, Anthony D, et al. A reengineered hospital discharge program to decrease rehospitalization: a randomized trial. Ann Intern Med. 2009;150:178-87.

11. Williams MV, Coleman E. BOOSTing the hospital discharge. J Hosp Med. 2009;4:209-10.

12. Buntin MB, Jain SH, Blumenthal D. Health information technology: laying the infrastructure for national health reform. Health Aff (Millwood). 2010;29:1214-9.

13. Dalal AK, Poon EG, Karson AS, Gandhi TK, Roy CL. Lessons learned from implementation of a computerized application for pending tests at hospital discharge. J Hosp Med. 2011;6:16-21.

14. Diem SJ, Prochazka AV, Meyer TJ, Fryer GE. Effects of a postdischarge clinic on housestaff satisfaction and utilization of hospital services. J Gen Intern Med. 1996;11:179-81.

15. Chaudhry SI, Mattera JA, Curtis JP, et al. Telemonitoring in patients with heart failure. N Engl J Med. 2010;363:2301-9. 